\title{
WE-Harvest: A Wearable Piezoelectric-Electromagnetic Energy Harvester
}

\author{
Rawnak Hamid \\ Electrical and Computer Systems \\ Engineering, Monash University, \\ Clayton, Melbourne VIC (3800), \\ Australia
}

\author{
Ali Mohammadi \\ Electrical and Computer Systems \\ Engineering, Monash University, \\ Clayton, Melbourne VIC (3800), \\ Australia.
}

\author{
Mehmet Rasit Yuce \\ Electrical and Computer Systems \\ Engineering, Monash University, \\ Melbourne, VIC (3800), Australia \\ mehmet.yuce@monash.edu
}

\begin{abstract}
Wearable electronics require a sustainable electrical power supply to operate. Energy harvesting techniques can be used to convert available nonelectrical energy sources into electrical energy. This paper presents WE-Harvest, a new wearable energy harvesting system that combines the piezoelectric and electromagnetic energy harvesters for wearable devices. Regular human body motions, such as moving the arm, provide the input vibrations. A two-stage modified Dickson multiplier is used to step up the output voltage. The experimental results demonstrate that the combined topology enhances the power transfer efficiency. The dependence of energy harvester output on the load and input frequency has also been investigated.
\end{abstract}

\section{Keywords}

Piezoelectric energy harvesting, magnetic energy harvesting, wearable energy harvesting, wearable sensors and devices.

\section{INTRODUCTION}

Wireless and portable applications provide convenience and new capabilities specifically in wireless sensors for health monitoring and wearable electronics [1]. However, the required electric power is supplied by batteries, which should be maintained frequently by changing or recharging. Chemical waste material of batteries is also an environmental threat. Recent advances in low power electronics have reduced the required electrical power for operating the sensors to several microwatts [2]. Hence, the energy generated by harvesting from ambient sources like heat, solar energy, wind, vibration and radio frequency waves can be used to power such devices [3-5].

The ubiquitous presence of vibration energy sources in our daily lives provide promising energy sources that can be harvested by micro power generators [6]. The focus of this work is on harvesting energy from low frequency vibrations like human motions. There are challenges involved due to the low repetition rates $(1-30 \mathrm{~Hz})$, large-amplitude, aperiodicity, predominantly inconsistent and time-varying vibration signatures of the natural motions [7][8]. Mechanical vibration energy can be extracted using mechanical-to-electrical transducers. Vibration-based power generators convert the mechanical energy into electrical energy in the form of alternative current (AC). The AC power is rectified to produce a stable DC (direct current) power using a power conditioning circuit since microelectronic devices and rechargeable batteries require a DC power supply. Therefore the performance of energy harvesting systems depends on both the transducer and power conditioning electronics. The operation principle of the proposed system, the design procedure and experimental results are described in sections II, III and IV, respectively.

\section{VIBRATION ENERGY HARVESTING METHODS}

In inertial energy harvesting techniques, the mechanical energy available in vibrating masses is converted to electrical energy. These techniques include piezoelectric, electro-magnetic and electrostatic transductions. Some of these techniques have been applied to energy harvesting applications on the human body [8][11]. In piezoelectric energy harvesting, mechanical stress and strain results in structural deformation caused by human motion and movements, which is then converted to electrical energy [8]. The electromagnetic induction is based on relative motions between a magnetic field and a coil, which causes electric current flow in the coil [9]. The amount of energy that can be harvested depends on the strength of the magnetic field, the velocity of the relative motion and the number of turns in the coil. Capacitive changes of the vibration-dependent capacitors are used for electrostatic energy harvesting. Mechanical energy is converted to electrical energy when vibrations move the plates initially charged [10][11]. A separate voltage source is needed for pre-charging the capacitor plates in electrostatic energy harvesters, which adds more complication in wearable applications.

Most of energy harvesting systems is optimized to harvest energy from one of available sources on the body. Electromagnetic energy harvesters can supply higher output currents, while the piezoelectric devices generate higher voltages. A combination of piezoelectric and electromagnetic techniques is presented in this work to improve the power generation efficiency for wearable energy harvesting systems.

\section{PROPOSED ENERGY HARVESTER}

Typically energy harvesting systems consist of a transducer that converts non-electrical energy to electrical energy and a power conditioning circuit that converts the alternative current (AC) to direct current (DC) and adjusts the voltage level to the corresponding load. The functional block diagram in Figure 1 
shows the operation principle of the energy harvester presented in this work. The proposed structure is designed to combine the electromagnetic induction and piezoelectric effect to convert the mechanical vibrations to electrical energy. The transducer and conditioning circuits for each technique are introduced next.

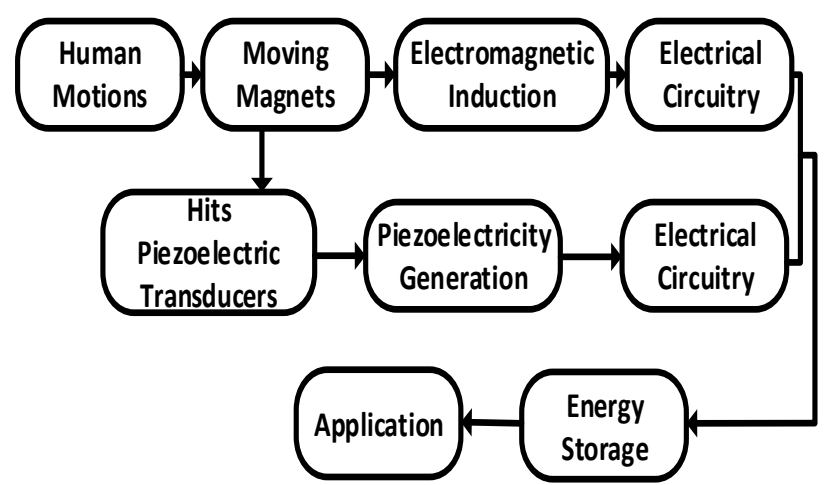

Figure 1:Functional block diagram of the energy harvesting device

\subsection{Transducer Design}

The mechanical structure of the proposed energy harvesting transducer is presented schematically in Figure 2, which features a pair of magnets that can slide freely inside the housing when vibrated. The copper wire is wound around the housing to convert the magnetic field changes initiated by the magnet motions to electric current. The piezoelectric transducers are fixed to the opposite walls of the housing. Human motions provide the vibration, which is needed to move the magnets back and forth, inducing current by electromagnetic induction. Simultaneously, the magnets hit the piezoelectric transducers when they reach the housing walls. Kinetic energy of the magnets applies a mechanical force to the piezoelectric layers that can be converted to electrical energy.

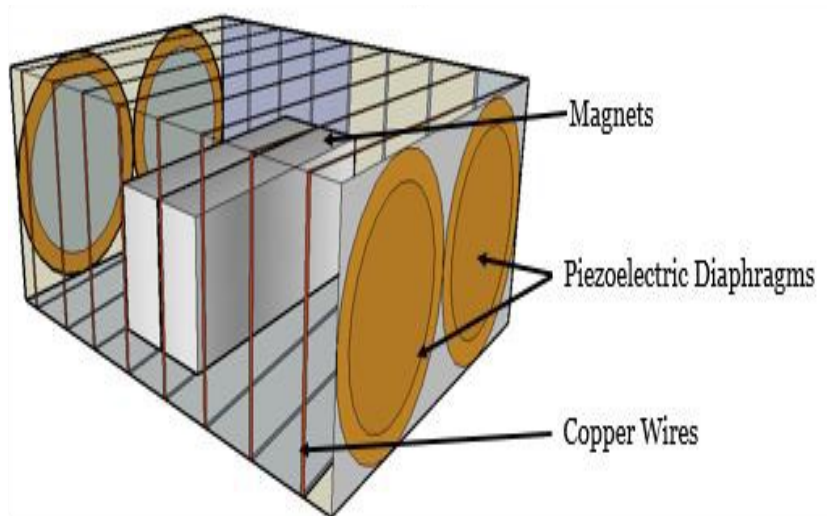

Figure 2: Schematic of the device

Four PZT (Lead Zirconate Titanate) diaphragms are electrically connected in parallel and mechanically fixed on a thin polycarbonate sheet at each side of the housing to improve the total amount of energy harvested by the PZT transducers. The parallel configuration enhance the electrical power since it increases the total output current while keeping the voltage constant. Using more than two layers of diaphragms increases the distance between the top and bottom layers and avoids the bottom layers receive enough pressure. The two sets of PZT diaphragms on opposite walls are also connected in parallel.

\subsection{Power Conditioning Circuitry}

A stable DC current is required in order to supply electrical power to the electronics or to charge batteries and super capacitors. Since the output power generated by both mechanisms is AC, they need to be rectified using suitable electrical circuitry. Off-theshelf integrated circuits such as LTC3588-1 can be used to rectify the output voltages from the piezoelectric transducers [12].

The amplitude of the voltage output from electromagnetic harvester is not large enough to overcome the rectifier threshold voltage. Therefore, two cascaded voltage doubler stages are employed in the proposed system to enhance and rectify the output voltage. The cascaded stage can generate four times larger output. Figure 3 shows the two stage Dickson multiplier schematic.

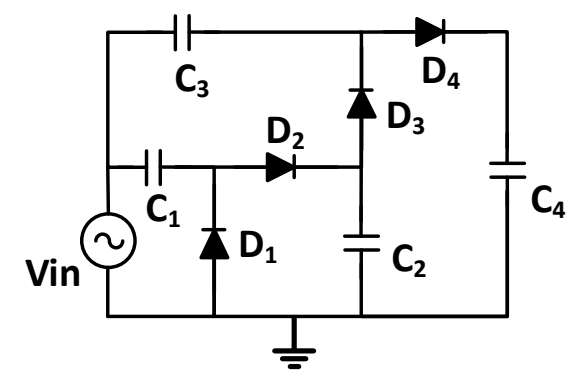

Figure 3: Two-stage Dickson multiplier

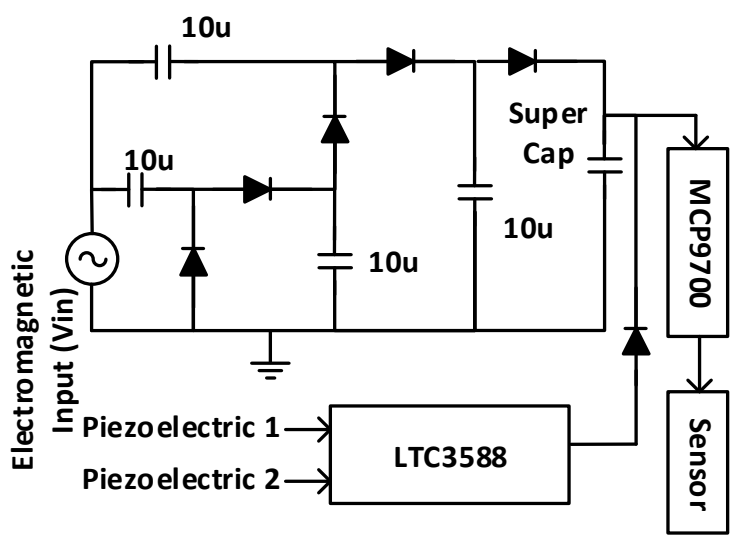

Figure 4: Power conditioning circuit, LTC3588 and MPC9700 are an off-the-shelf rectifier and a Linear Active Thermistor, respectively

The operation of voltage doubler in Figure 3 follows a certain sequence of cycles. During the input negative half cycle, D1 will charge the $\mathrm{C} 1$ voltage up to Vin. In the positive half cycle, the top plate of $\mathrm{C} 1$ is clamped to $2 \mathrm{Vin}$. D1 is then turned off while D2 turns on and charge cycle for $\mathrm{C} 2$ starts. In the next negative half cycle D3 turns on and charges $\mathrm{C} 3$ to $3 \mathrm{Vin}$, and in the positive half cycle the top plate of $\mathrm{C} 3$ is clamped to $4 \mathrm{Vin}$. The combination of D2, C2, D4 and C4 act as a peak detector and smooth the ripple without increasing the multiplication. Tantalum capacitors and Schottky diodes are used to reduce the losses in the circuit. The 
diodes maximum voltage drop is $400 \mathrm{mV}$ at $10 \mathrm{~mA}$ forward current [13]. A schematic of the overall power conditioning circuitry used to combine the electromagnetic and piezoelectric harvesters is shown in Figure 4. The output voltage of piezoelectric transducers is rectified by a power electronic module which includes a bridge rectifier and a buck converter (LTC3588). A large super capacitor is used to store the energy generated by the two harvesters. The reverse leakage of current from each harvester to the other is avoided by using diodes between harvester outputs and large capacitor. A typical application for the energy harvester is to power up low-power sensors such as temperature sensor (MCP9700). The final output of power conditioning circuitry is used to supply the sensor as a proof of concept. Further details are addressed in the experiment section.

\section{EXPERIMENTS}

Figure 5 shows the prototype designed in this work for integrating electromagnetic and piezoelectric energy harvesting techniques.

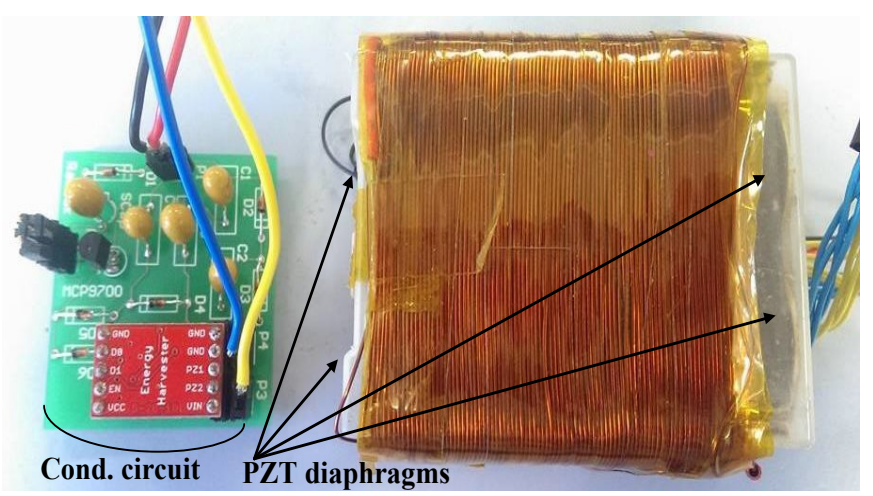

Figure 5: Prototype wearable piezoelectric-electromagnetic energy harvester.

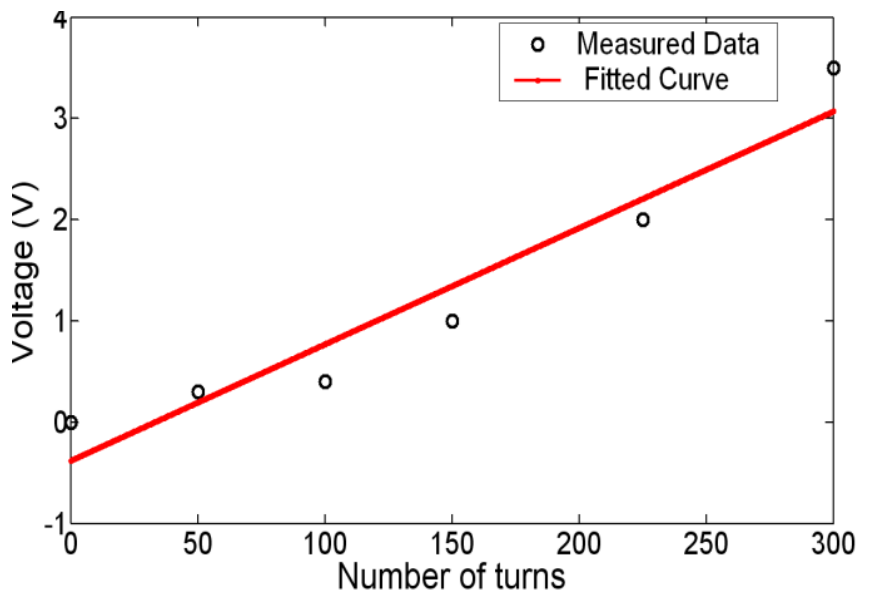

Figure 6: Plot showing the linear relationship between number of turns of coil and the output peak to peak voltage

The device was shaken manually to produce mechanical input. Experiments were repeated for each technique, individually, and their combination to show the improvements after combining the two techniques.

\subsection{Electromagnetic Energy Harvester}

The voltage generated at the electromagnetic energy harvester output is proportional to the number of turns in the coil. The result of experiments carried out for different number of turns in the proposed structure is illustrated in Figure 6. The energy harvester output voltage is enhanced by a Dickson multiplier. The number of multiplier stages required to achieve the desired output voltage $(\sim 3.3 \mathrm{~V})$ is found by simulations as shown in Figure 7 . The sensor that is used in this work, as an example application, has an input impedance of $280 \mathrm{~K} \Omega$. Therefore, the output was measured using a range of different load resistances ranging from $15 \mathrm{k} \Omega$ to $300 \mathrm{k} \Omega$. Figure 8 demonstrates the output voltage with respect to the load resistances.

\subsection{Piezoelectric Energy Harvester}

The output voltage of a piezoelectric energy harvester is proportional to the frequency of vibration in addition to its amplitude. The frequency dependence can be explained by the capacitive structure of the piezoelectric device. Short time periods of high frequency inputs avoid the capacitive discharge on these devices. In the proposed system, a single piezoelectric diaphragm $(\mathrm{PZT})$ is vibrated at varying frequencies ranging from $1 \mathrm{~Hz}-5 \mathrm{~Hz}$ and the corresponding output voltage is recorded (See Table 1.).

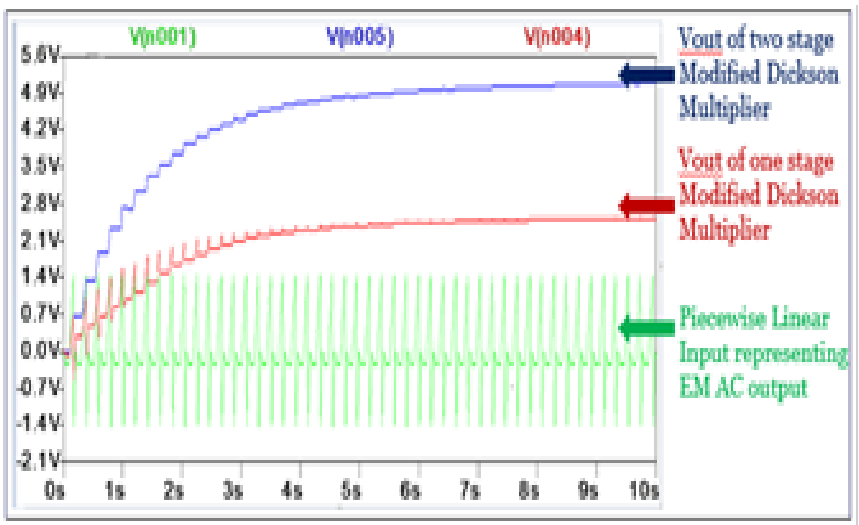

Figure 7. Simulation result for modified Dickson Multiplier

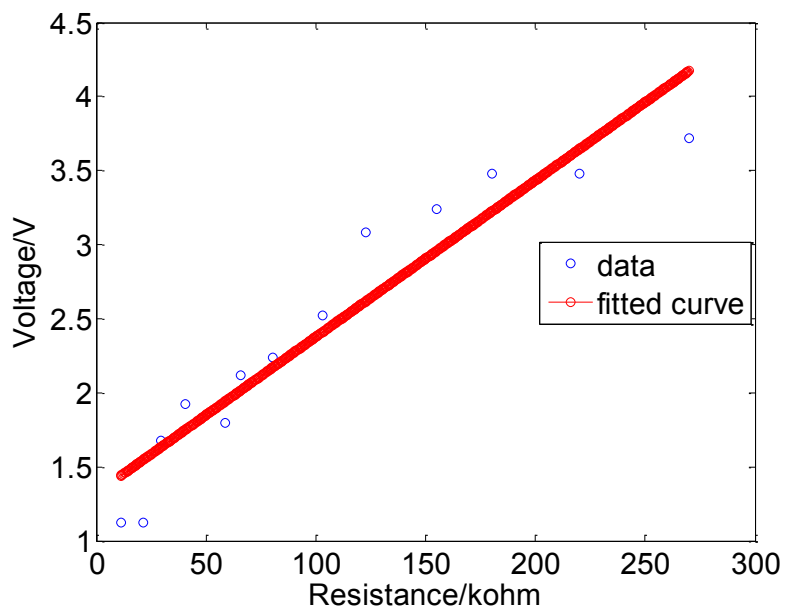

Figure 8: Plot showing the output voltage across varying load resistors 
Table 1. RMS voltage obtained from a Piezoelectric diaphragm in different frequencies.

\begin{tabular}{|c|c|c|c|c|c|}
\hline Frequency (Hz) & 1 & 2 & 3 & 4 & 5 \\
\hline RMS Voltage (V) & 1.6 & 2.3 & 3 & 3.1 & 3.2 \\
\hline
\end{tabular}

The current output from a single PZT was about $1 \mu \mathrm{A}$, which is very low. Connecting piezoelectric transducers in parallel increases their current output while keeping the output voltage constant. The transducer AC output voltage is rectified by an offthe-shelf circuit (LTC3588), which regulates the output voltage to $3.3 \mathrm{~V}$. The rise time of a load capacitor charging curve was measured in order to evaluate the systems performance. A plot showing the comparison of the rise time of the different combinations of piezoelectric diaphragms is illustrated in Figure 9, which shows that with increased number of PZT diaphragms, the time to charge the capacitor decreases. The piezoelectric diaphragms are generally modeled as current sources. Therefore parallel configuration of piezoelectric diaphragms is expected to increase the power output and thus lead to charging the capacitor faster. The approximate input frequency is about $5 \mathrm{~Hz}$ in the experiments.

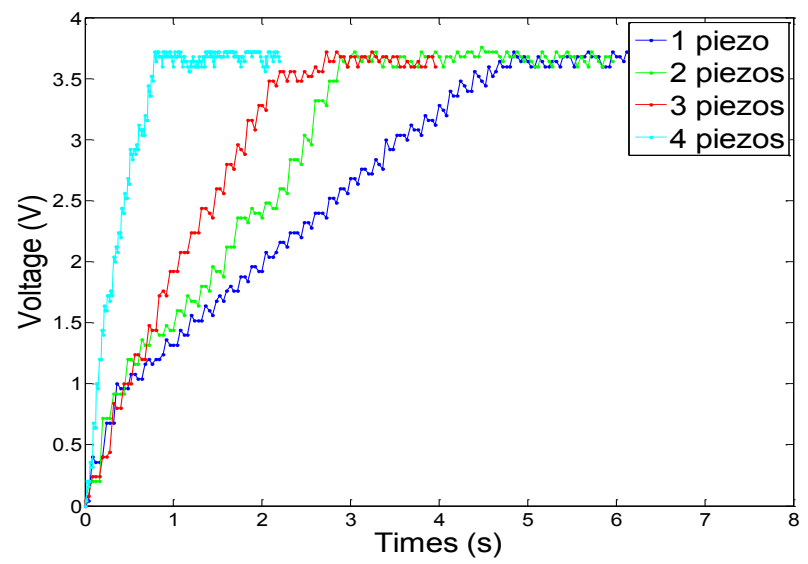

Figure 9: Plot comparing the rise time to charge a $22 \mu \mathrm{F}$ capacitor using different combinations of piezoelectric diaphragms

\subsection{Electromagnetic and Piezoelectric Coupled Energy Harvester}

In the integrated system, capacitors from the individual harvesters discharge into a single energy storage device through diodes. Thus they need to have comparable output voltage since the coupled device follows the output voltage of the harvester having lower output. The two individual energy harvesters and the coupled device were used to charge $10 \mu \mathrm{F}$ capacitors.

Figure 10 illustrates that the coupled energy harvester takes less time to charge the same capacitor due to the increased output power harvested. Table 2 compares individual and coupled energy harvesting techniques. Measurement results show that the coupled energy harvester takes less than half the time to generate the same power as the individual harvesters. Hence it can be said that a coupled energy harvester is more efficient than the ones using only one harvesting technique.

\section{CONCLUSION}

This paper presents an energy harvesting technique that harvests energy from low frequency vibrations like human motion and stores the harvested energy to power wearable devices. Electromagnetic and piezoelectric energy harvesting techniques were combined, and the resulting energy was stored in a capacitor. It is shown that the combined system is capable of producing higher energy level for a certain amount of mechanical input. It was able to generate $45 \mu \mathrm{W}$ of power when the frequency of vibration was about $3 \mathrm{~Hz}$. The proposed technique was tested to power a temperature sensor, MCP9700, with a memory to store values.

\section{Acknowledgment}

Mehmet R. Yuce's work was supported by Australian Research Council Future Fellowships Grant FT130100430.

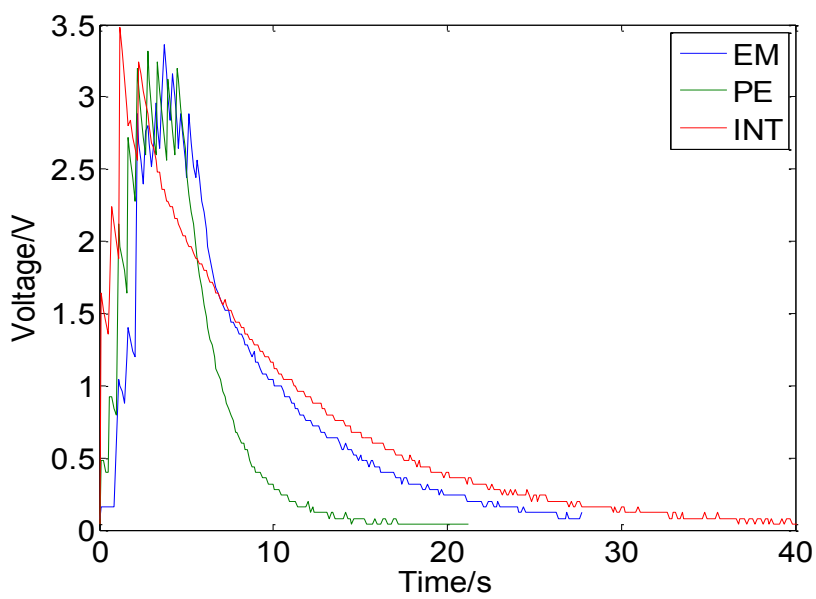

Figure 10: The output voltage from the energy harvesting device along with the individual methods, Electromagnetic, Piezoelectric and Integrated approaches.

Table 2. Comparison of individual and coupled energy harvesters

\begin{tabular}{|l|l|l|}
\hline Energy Harvester & $\begin{array}{l}\text { Output } \\
\text { Power } \\
(\mu \mathrm{W})\end{array}$ & Rise-time (s) \\
\hline Electromagnetic & 43.8 & 3.7 \\
\hline Piezoelectric & 42.8 & 2.8 \\
\hline Coupled & 44.8 & 1.2 \\
\hline
\end{tabular}

\section{REFERENCES}

[1] M. R. Yuce, "Implementation of wireless body area networks for healthcare Systems," Sensors \& Actuators: A. Physical, vol. 162, pp. 116-129, July 2010.

[2] S. Chalasani and J. M. Conrad, "A survey of energy harvesting sources for embedded systems," in Proc. IEEE Southeastcon, 2008, pp. 442-447. 
[3] J. Yoo, H-J. Yoo, "Emerging low energy wearable body sensor networks using patch sensors for continuous healthcare applications," Engineering in Medicine and Biology Society (EMBC), Annual International Conference of the IEEE , pp.6381,6384, Aug. 31 -Sept. 42010.

[4] M. H. Ghaed, et al., "Circuits for a cubic-millimeter energyautonomous wireless intraocular pressure monitor," IEEE Trans. Circuits Syst. I, vol. 60, no. 12, pp. 3152-3162, December 2013.

[5] J. P. Fleurial, G J Snyder, J. A. Herman, et al., "Miniaturized thermoelectric power sources," in Proc. Intersociety Energy Conversion Engineering Conference, Vancouver, BC, Canada, 1999.

[6] S. W. Liu, S. W. Lye, and J.M. Miao, "Sandwich structured electrostatic/electrets parallel-plate power generator for low acceleration and low frequency vibration energy harvesting," in Proc. IEEE 25th International Conference on Micro Electro Mechanical Systems (MEMS), pp. 1277-1280, 2012.

[7] N. Kong, D. S. Ha, A. Erturk, and D.J. Inman, "Resistive impedance matching circuit for piezoelectric energy harvesting," Journal of Intelligent Material Systems and Structures, vol. 21, pp. 1293-1302, September 2010.
[8] E. Shahhaidar, et al., "Piezoelectric and electromagnetic respiratory effort energy harvesters," 35th Annual International Conference of the IEEE Engineering in Medicine and Biology Society (EMBC), pp.3439-3442, 3-7 July 2013.

[9] B. J. Bowers and D. P. Arnold, "Spherical, rolling magnet generators for passive energy harvesting from human motion," Journal of Micromechanics and Microengineering, vol. 19, no. 9, August 2009.

[10] D. Zhu, "Vibration energy harvesting: machinery vibration, human movement and flow induced vibration," chapter 2,Sustainable Energy Harvesting Technologies - Past, Present and Future, Y. K. Tan, Ed., ed, In-Tech, 2011.

[11] Y. Zhu, S. O. R. Moheimani, M. R. Yuce, " A 2-DOF MEMS ultrasonic energy harvester," IEEE Sensors Journal, vol 11, pp. 155-161, January 2011.

[12] Piezoelectric Energy Harvesting Power Supply, LTC3588-1. DataSheet.: http://www.linear.com/product/LTC3588-1.

[13] P. Nintanavongsa, et al., "Design optimization and implementation for RF energy harvesting circuits," IEEE J. Emerging and Selected Topics in Circuits and Systems, vol. 2, pp. 24-33, March 2012. 